OPEN ACCESS

Edited by: Massimo Breccia, Sapienza Università di Roma, Italy

Reviewed by: Francesco Buccisano, Università degli Studi di Roma Tor Vergata, Italy Naval Daver,

University of Texas MD Anderson Cancer Center, United States

*Correspondence: Carmen Mariana Aanei cmaanei@gmail.com

Specialty section: This article was submitted to Hematology Oncology, a section of the journal Frontiers in Oncology

Received: 15 January 2018 Accepted: 28 March 2018 Published: 12 April 2018

Citation:

Picot T, Aanei CM, Flandrin Gresta P, Noyel $P$, Tondeur $S$, Tavernier Tardy E,

Guyotat $D$ and Campos Catafal $L$ (2018) Evaluation by Flow Cytometry of Mature Monocyte Subpopulations for the Diagnosis and Follow-Up of Chronic Myelomonocytic Leukemia. Front. Oncol. 8:109. doi: 10.3389/fonc.2018.00109

\section{Evaluation by Flow Cytometry of Mature Monocyte Subpopulations for the Diagnosis and Follow-Up of Chronic Myelomonocytic Leukemia}

\author{
Tiphanie Picot ${ }^{1}$, Carmen Mariana Aanei ${ }^{1 *}$, Pascale Flandrin Gresta ${ }^{1}$, Pauline Noyel', \\ Sylvie Tondeur ${ }^{1}$, Emmanuelle Tavernier Tardy ${ }^{2}$, Denis Guyotat ${ }^{2}$ and Lydia Campos Catafal ${ }^{1}$ \\ 'Laboratoire d'Hématologie, CHU de Saint-Etienne, Saint-Etienne, France, ${ }^{2}$ Département d'Hématologie et Thérapie \\ Cellulaire, Institut de Cancérologie Lucien Neuwirth, Saint-Etienne, France
}

Chronic myelomonocytic leukemia (CMML) is a myelodysplastic/myeloproliferative neoplasm, characterized by persistent monocytosis and dysplasia in at least one myeloid cell lineage. This persistent monocytosis should be distinguished from the reactive monocytosis which is sometimes observed in a context of infections or solid tumors. In 2015, Selimoglu-Buet et al. observed an increased percentage of classical monocytes $\left(\mathrm{CD} 14^{+} / \mathrm{CD}^{-} 6^{-}>94 \%\right)$ in the peripheral blood (PB) of CMML patients. In this study, using multiparametric flow cytometry (MFC), we assessed the monocytic distribution in PB samples and in bone marrow aspirates from 63 patients with monocytosis or CMML suspicion, and in seven follow-up blood samples from CMML patients treated with hypomethylating agents (HMA). A control group of 12 healthy age-matched donors was evaluated in parallel in order to validate the analysis template. The CMML diagnosis was established in 15 cases in correlation with other clinical manifestations and biological tests. The MFC test for the evaluation of the repartition of monocyte subsets, as previously described by Selimoglu-Buet et al. showed a specificity of $97 \%$ in blood and $100 \%$ in marrow samples. Additional information regarding the expression of intermediate $\mathrm{MO} 2$ monocytes percentage improved the specificity to $100 \%$ in blood samples allowing the screening of abnormal monocytosis. The indicative thresholds of CMML monocytosis were different in PB compared to BM samples (classical monocytes $>95 \%$ for PB and $>93 \%$ for BM). A decrease of monocyte levels in $\mathrm{PB}$ and $\mathrm{BM}$, along with a normalization of monocytes distribution, was observed after treatment in 4/7 CMML patients with favorable evolution. No significant changes were observed in 3/7 patients who did not respond to HMA therapy and also presented unfavorable molecular prognostic factors at diagnosis (ASXL1, TET2, and IDH2 mutations). Considering its simplicity and robustness, the monocyte subsets evaluation by MFC provides relevant information for CMML diagnosis.

Keywords: chronic myelomonocytic leukemia, flow cytometry, monocytic subpopulations, peripheral blood monocytosis, next-generation sequencing, karyotype 


\section{INTRODUCTION}

Chronic myelomonocytic leukemia (Chronic myelomonocytic leukemia) is a clonal malignant hematological disorder characterized by monocytosis and myeloid dysplastic features (1). According to the World Health Organization (WHO) classification, it has been recognized as a distinct entity from myelodysplastic syndromes and grouped with other rare myeloid malignancies that combine myeloproliferative and dysplastic traits (2). Nowadays, the biological diagnosis of CMML is based on the presence of an increased level of monocytes in peripheral blood (PB) $\left(>10 \%,>1 \times 10^{9} / \mathrm{L}\right.$, for at least 3 months $)(3,4)$. However, it may be difficult to discriminate CMML from reactive monocytosis $\left(>1 \times 10^{9} / \mathrm{L}\right)$ or prefibrotic myelofibrosis using only these criteria. For these reasons, multiparametric flow cytometry (MFC) was tested to evaluate the phenotypic profile in bone marrow (BM) or blood samples to find specific changes related to CMML. The study conducted by Shen et al. in 118 CMML patients, promotes integration of MFC data with other clinical and biological tools in the diagnosis of CMML (5). They evaluated all myeloid compartments (immature and mature) in BM aspirates and found alterations in granulocytic maturation in more than two-thirds of CMML patients (5). Likewise, they observed an increased expression of mature myelomonocytic markers on CD34 ${ }^{+}$myeloblasts, including CD64, CD15, and rarely, CD11b, in a small subset of CMML cases (20 of 118, 17\%) (5). However, the authors observed that immunophenotypic changes in monocytes, using the criteria established by International Workshops from the European LeukemiaNet Working Group, are not specific for CMML or other MDS settings, but make the neoplastic process visible if present (5).

A startling idea suggests that monocyte subpopulations in blood are a "mirror of disrupted homeostasis and disease" (6). Therefore, it is worth evaluating for the mature monocyte compartment. Three subpopulations of mature monocytes (classical monocytes, noted hereafter as MO1; intermediate monocytes, $\mathrm{MO} 2$ and nonclassical, MO3) were described by the Nomenclature Committee of the International Union of Immunological Societies (NCIUIS) (7). This subdivision was validated using gene expression profiling (8-10). Recently, Selimoglu-Buet et al. demonstrated, in CMML patients, an increase in the fraction of MO1 $\mathrm{CD} 14^{+} / \mathrm{CD} 16^{-}$monocytes ( $>94 \%$ for a specificity of $95.1 \%$ and a sensitivity of $90.6 \%)$ at the expense of $\mathrm{MO} 2\left(\mathrm{CD} 14^{+} / \mathrm{CD} 16^{+}\right)$and MO3 (CD14 $\left.{ }^{\text {+low }} / \mathrm{CD} 16^{+}\right)$cell fractions (11).
The aim of this study was to evaluate by MFC, the monocyte subsets, using Selimoglu-Buet test in patients showing PB monocytosis and clinical suspicion of CMML compared to "healthy" subjects and to patients carrying other hematological diseases, in order to validate this test for routine practices. The test shows excellent sensitivity and specificity for CMML diagnosis, allowing exclusion of reactive monocytosis, and permitting monitoring of CMML treatment.

\section{MATERIALS AND METHODS}

\section{Study Group}

The study group consisted of 63 cases of monocytosis or CMML suspicion analyzed in real time, which were referred to the Institut de Cancérologie Lucien Neuwirth between March 2016 and December 2017 for diagnosis or follow-up after chemotherapy. The diagnosis of CMML was established according to the current WHO criteria (12) by a combination of clinical findings, morphologic evaluation of $\mathrm{PB}$, bone marrow specimens, conventional cytogenetic and molecular analysis. The "reactive" monocytosis was considered (1) in a context of transient monocytosis ( $<6$ months) or inflammatory disease, (2) when BM examination showed few or absent dysplastic signs, a normal karyotype and the absence of mutations by next-generation sequencing (NGS). The final distribution of the cases was CMML $(n=15)$, reactive monocytosis $(n=36)$, and hematological malignancies $(n=12)$, including chronic myeloid leukemia $(n=1)$, myelodysplastic syndromes $(n=2)$, acute myeloid leukemia $(n=4)$, multiple myeloma $(n=1)$, and myeloproliferative neoplasms $(n=4)$. In three patients with CMML and in one patient with reactive monocytosis, we evaluated both bone marrow and blood samples. In addition, monocytes subsets were evaluated in seven CMML samples ( $\mathrm{BM}, n=3$ or $\mathrm{PB}, n=4$ ) during the clinical follow-up. Detailed characteristics of these groups are shown in Table 1. Twelve age-matched healthy subjects were analyzed in order to evaluate the robustness of the gating strategy.

The "Comité de Protection des Personnes" (Independant Ethics Committee) Sud-Est 1 from University Hospital of SaintEtienne, France has reviewed and has given ethical approval for the study. All patients gave informed consent according to the institutional procedures.

TABLE 1 | Patients characteristics.






\section{MFC Staining and Analysis}

Multiparametric flow cytometry analysis of monocyte subsets was performed on whole $\mathrm{PB}$ and BM samples collected on EDTA. Erythrocyte lysis was performed using FACS lysing solution (BD Biosciences, CA, USA). Cell surface staining of $10^{6}$ cells was performed using a combination of antibodies (Table 2) for $15 \mathrm{~min}$ at room temperature, sheltered from light. At least 300,000 total events and 30,000 events in the monocyte subpopulation were acquired (FACS Canto II, BD Biosciences). Data were analyzed using Diva software version 6.1.3 (BD Biosciences). Cytometer settings were established conforming to EuroFlow procedures, and the instrument quality control was checked on a daily basis (13). The monocyte subsets were identified following an exclusion gating strategy as described by Selimoglu-Buet (11). Briefly, monocytes were gated on a CD45/SSC dot plot followed by the exclusion of T lymphocytes expressing CD2, of NK lymphocytes expressing CD2, CD16, and CD56, of memory B-lymphocytes $\mathrm{CD} 24^{+}$, of other residual contaminating cells CD14- and CD16-, and of granulocytes expressing CD16 ${ }^{\text {thigh }}$. Thereafter, the CD16 and CD14 markers were used to discriminate the subsets of mature monocytes (MO1 CD14 ${ }^{+} / \mathrm{CD}_{16}{ }^{-}$, conventional monocytes; $\mathrm{MO} 2 \mathrm{CD}_{1} 4^{+} / \mathrm{CD} 16^{+}$, intermediate monocytes; and $\mathrm{MO} 3$ $\mathrm{CD} 14^{\text {low }} / \mathrm{CD}_{16}{ }^{+}$, unconventional monocytes) (Figure 1). The distribution of monocytes in different subsets was reported as the percentage of the total monocytes. Furthermore, we assessed monocyte distribution stability by measuring MO1, MO2, and $\mathrm{MO} 3$ fractions in different time points: within 24 and $48 \mathrm{~h}$ after harvesting $(n=5)$.

\section{Morphologic Examination}

The light microscopic assessment of PB smears and BM aspirates evaluated after May-Grunwald-Giemsa staining was performed for each case by two experimented pathologists. The dysplastic changes were reported according to WHO recommendations (12).

\section{Next-Generation Sequencing (NGS)}

Genomic DNA samples were tested in 13/15 CMML patients, by NGS, using a custom designed myeloid panel, based on an AmpliSeq strategy (Life Technologies, CA, USA). The panel addressed 24 recurrently mutated genes in myeloid malignancies: ASXL1, CALR, CSF3R, DNMT3A, EZH2, FLT3, GATA2, IDH1, IDH2, JAK2, KIT, KRAS, NPM1, NRAS, PTPN11, RUNX1, SETBP1, SF3B1, SRSF2, TET2, TP53, U2AF1, and WT1. Libraries were constructed using the Ion AmpliSeq Library Kit v2.0 according to the manufacturer's instructions. Amplification of libraries, loading on 316 chip V2, was performed with the Ion Chief System ${ }^{\circledR}$, while sequencing was executed using the Ion $\mathrm{PGM}^{\circledR}$ machine (Life Technologies). Ion Reporter ${ }^{\circledR}$ and NextGENe $^{\circledR}$ v.2.3.4 (SoftGenetics, USA) software were used to perform bioinformatics analysis, including optimized signal processing, base calling, sequence alignment (hg19 reference), and variant analysis. Variants detected with a frequency of $2 \%$ or higher on both strands were considered as present. Sanger sequencing was performed for all patients to identify the ASXL1 c.1934dupG (p.G5646Wfs ${ }^{\star} 12$ ), as this mutation, occurring in a homopolymer region, is not detected with our NGS approach. Furthermore, all variants not referenced as mutational hotspots in international databases (Ensembl, Cosmic, IARC TP53), and detected with a VAF $>20 \%$, were confirmed by Sanger sequencing.

\section{Karyotype}

Bone marrow samples for cytogenetic analysis were obtained for $13 / 15$ CMML patients at the time of diagnosis. Karyotypes were analyzed after 24-h culture following standard procedures. The chromosomes were stained by R- and G-banding. At least 20 metaphases were analyzed. Results were interpreted and reported according to the International System for Human Cytogenetic Nomenclature (ISCN, 2013 and 2016) (14).

\section{Statistical Analysis}

The nonparametric Fisher's test was used to compare distributions between groups. Cut-offs were estimated in the learning cohort by maximizing the Youden index $(J=$ sensitivity + specificity -1$)$. These cut-offs were compared to the classical CD $14^{+} /$ CD16- monocyte count cut-off of $1 \times 10^{9} / \mathrm{L}$.

Analysis and figure plotting were performed with GraphPad Prism 5 software (GraphPad, CA, USA).

\section{RESULTS}

\section{Distribution of Monocyte Subsets}

The relative frequencies of monocyte subsets in the "healthy" group were $87.04 \% \pm 3.7 \mathrm{MO} 1,4.2 \% \pm 1.79 \mathrm{MO} 2$, and $8.7 \% \pm 3.98 \mathrm{MO} 3$ and in patients with reactive monocytosis $82.7 \% \pm 13.61 \mathrm{MO} 1,8.4 \% \pm 11.97 \mathrm{MO} 2$, and $8.6 \pm 6.41$ MO3 (Figure 2A). CMML cases demonstrated a significant increase in MO1 percentage $(p<0.001)$ compared to MO2 and $\mathrm{MO} 3$ subsets in $\mathrm{PB}(97.2 \% \pm 4.0 \mathrm{MO} 1,1.8 \% \pm 1.8 \mathrm{MO} 2$, and $0.9 \% \pm 2.3 \mathrm{MO} 3)$ and in $\mathrm{BM}(98.7 \% \pm 3.5 \mathrm{MO} 1,0.6 \% \pm 0.9$ $\mathrm{MO} 2$, and $0.5 \% \pm 2.9 \mathrm{MO} 3$ ) (Figure $2 \mathrm{~A}$ ). In CMML patients,

TABLE 2 | List of antibodies used for multiparametric flow cytometry.

\begin{tabular}{|c|c|c|c|c|c|}
\hline Antigen & Antibodies & Clone (isotype) & Fluorochrome & Company & Reference \\
\hline CD45 & Mouse anti-human CD45 & HI30 (mouse IgG1, к) & Pacific orange (V-500) & Beckton Dickinson & 560777 \\
\hline CD24 & Mouse anti-human CD24 & ML5 (mouse IgG2a, к) & $\mathrm{PE}$ & Beckton Dickinson & 555428 \\
\hline CD2 & Mouse anti-human CD2 & TS1/8 (mouse lgG1, $\kappa)$ & Pacific blue (V-450) & Biolegend & 309216 \\
\hline CD14 & Mouse anti-human CD14 & MpP9 (mouse BALB/c lgG2b, к) & APC-H7 & Beckton Dickinson & 641394 \\
\hline CD16 & Mouse anti-human CD16 & CLB-FcR-gran/1, 5D2 (mouse lgG2a) & FITC & PeliCluster Sanquin & M1389 \\
\hline CD56 & Mouse anti-human CD56 & N901, NKH-1 (mouse IgG1) & PE-CY7 & Beckman Coulter & A21692 \\
\hline
\end{tabular}





FIGURE 1 | Gating strategy used in multiparametric flow cytometry analysis to detect different subsets of monocytes. Doublets exclusion on FSC-A/FSC-H dot plot (A). Live cells on FSC-A/SSC-A (B). Monocytes identification as CD45+/SSC intermediate cells (C). Invert gate and exclusion of cells expressing CD24+, granulocytes expressing CD16 ${ }^{\text {thigh }}$, and lymphocytes expressing CD56 ${ }^{+}$and CD2+ (D-G). Exclusion of residual cells CD14- CD16- (H). Characterization of human monocyte subsets into $\mathrm{CD}_{14}{ }^{+} / \mathrm{CD} 16^{-}$(MO1, conventional), CD14+/CD16+ (MO2, intermediate), and CD14 low/CD16 ${ }^{+}$(MO3, unconventional) subsets (I). Cell hierarchy (J). 
A “Healthy Subject"

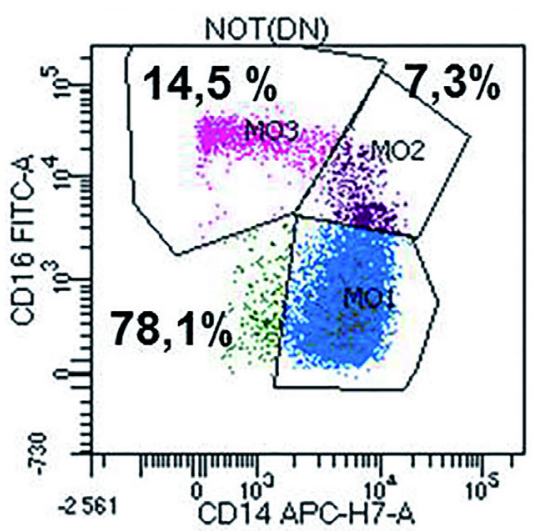

Non CMML

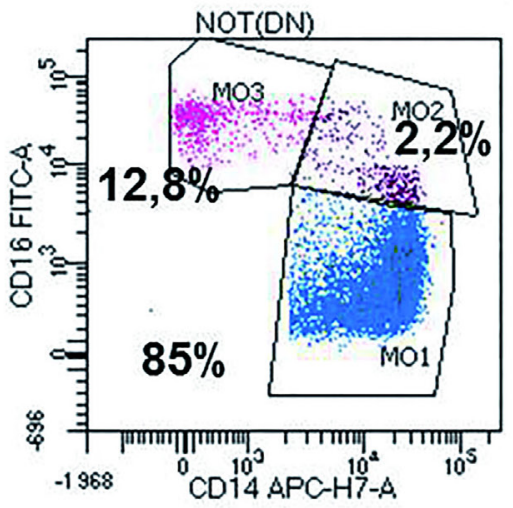

Reactive monocytosis

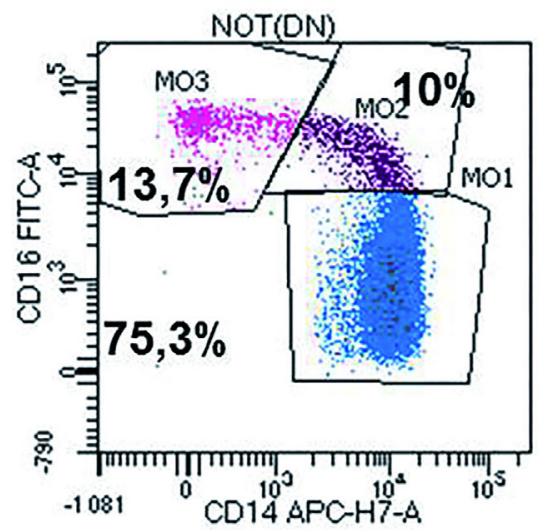

CMML

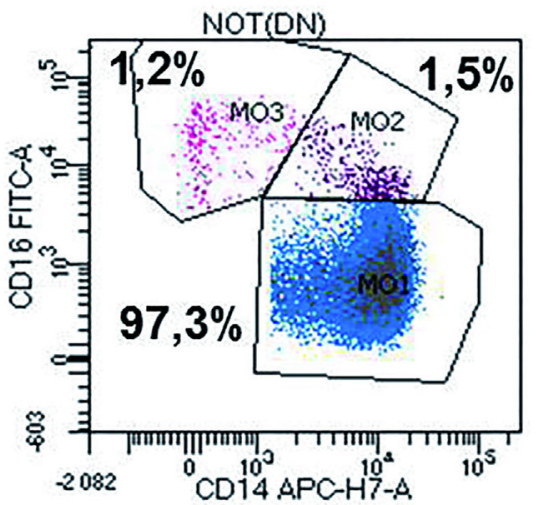

B

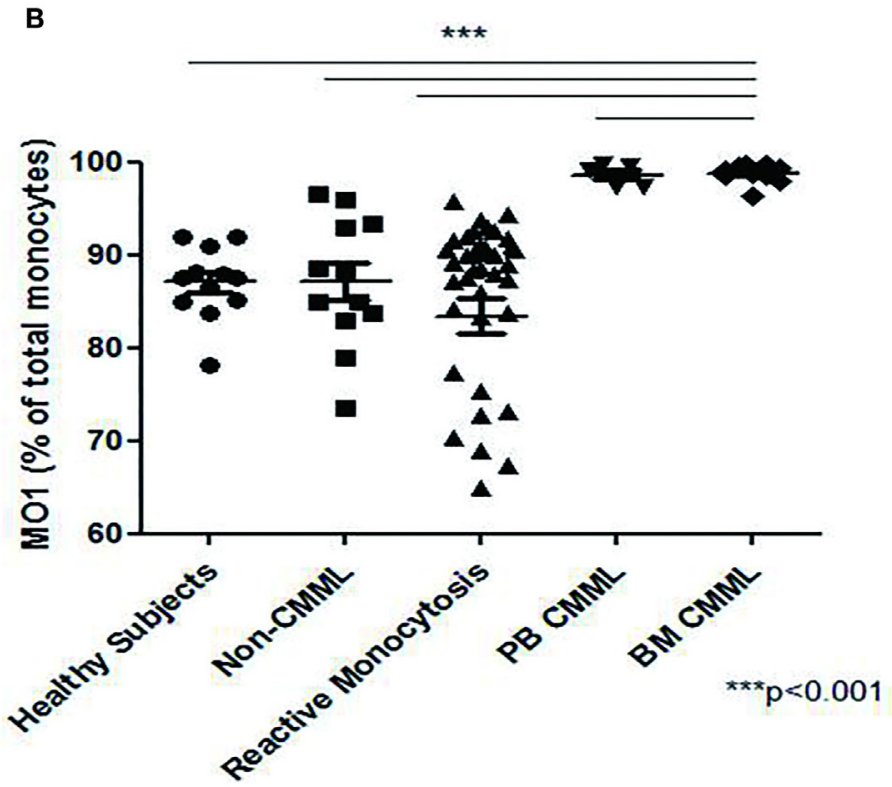

FIGURE 2 | Repartition of monocytes subsets in different groups of cases. Monocyte subset repartition in distinct groups [ $n=12$ "healthy subjects," $n=15$ chronic myelomonocytic leukemia (CMML), $n=36$ reactive monocytosis, $n=12$ hematological diseases or non-chronic myeloid leukemia (CMML) $(n=1)$, MDS ( $n=2$ ), AML $(n=4)$, myeloma $(n=1)$, and myeloproliferative syndromes $(n=4)$ ]. For each group, the monocytes subsets expressed as percentage of total monocytes are displayed (A). Relative frequencies of the MO1 subset in different groups $\left(n=12\right.$ "healthy subjects," $n=12$ non-CMML, $n=36$ reactive monocytosis $>1 \times 10^{9}, n=5$ peripheral blood CMML, and $n=10$ bone marrow CMML) (B). Fisher test was performed in order to compare each group of cases with healthy subjects. ${ }^{\star \star \star} p<0.001$. 


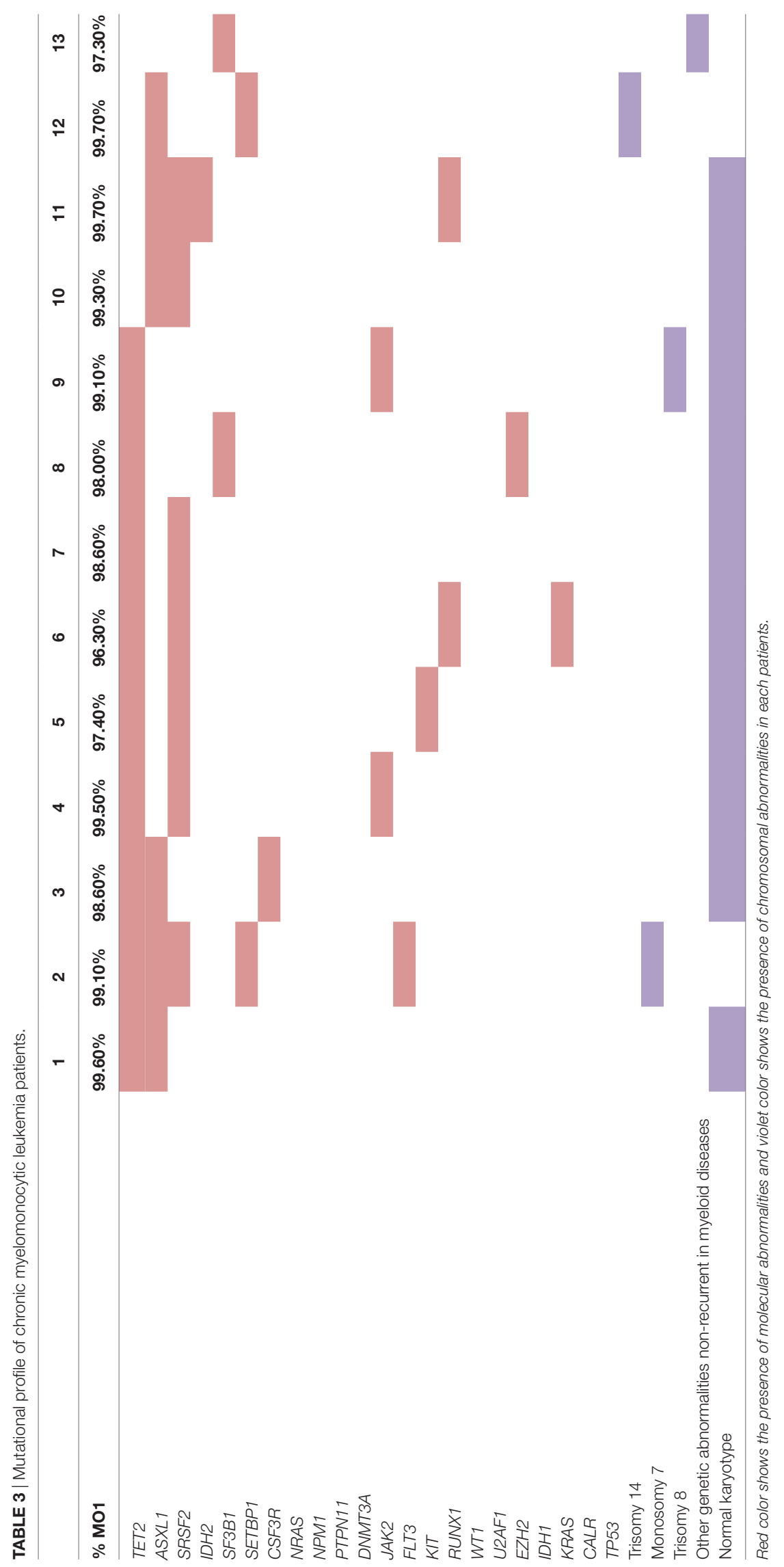


dysplastic signs of one or more BM myeloid cells were observed, along with the presence of clonal genetic abnormalities (the most frequent mutations were ASXL1: $n=6 / 13$ patients, SRSF2: $n=7 / 13$ patients, and TET2: $n=9 / 13$ patients) (Table 3 ). $4 / 13$ CMML patients harbored an abnormal karyotype: three patients presented recurrent alterations found in myeloid malignancies (monosomy 7, trisomy 8, and trisomy 14), whereas the fourth one presented genetic abnormalities which were non-recurrent in myeloid diseases.

Patients carrying other myeloid malignancies showed a similar pattern of monocyte distribution to those having reactive monocytosis $(87.1 \% \pm 7.07 \mathrm{MO} 1,5.4 \% \pm 5.79 \mathrm{MO} 2$, and $7 \% \pm 4.65 \mathrm{MO} 3$ ) (Figure 2B).
Interestingly, we observed changes in $\mathrm{MO} 1, \mathrm{MO} 2$, and $\mathrm{MO} 3$ distribution in two CMML samples when MFC was performed at 24 and $48 \mathrm{~h}$ after harvesting. While MO1 percentage was consistent with CMML at $24 \mathrm{~h}(>96 \%)$, the analysis at $48 \mathrm{~h}$ showed a monocyte profile that was similar to that observed in reactive monocytosis (Figure 3B).

\section{The Contribution of MFC in Detection of CMML Monocytosis}

The statistical analysis showed that the cut-off percentage of MO1 suggestive of CMML in PB was 95\% (97\% specificity, 100\% sensitivity, $p<10^{-4}, \chi^{2}$ test, Youden index $\left.=0.97\right)$ and $93 \%$ in

\section{A}

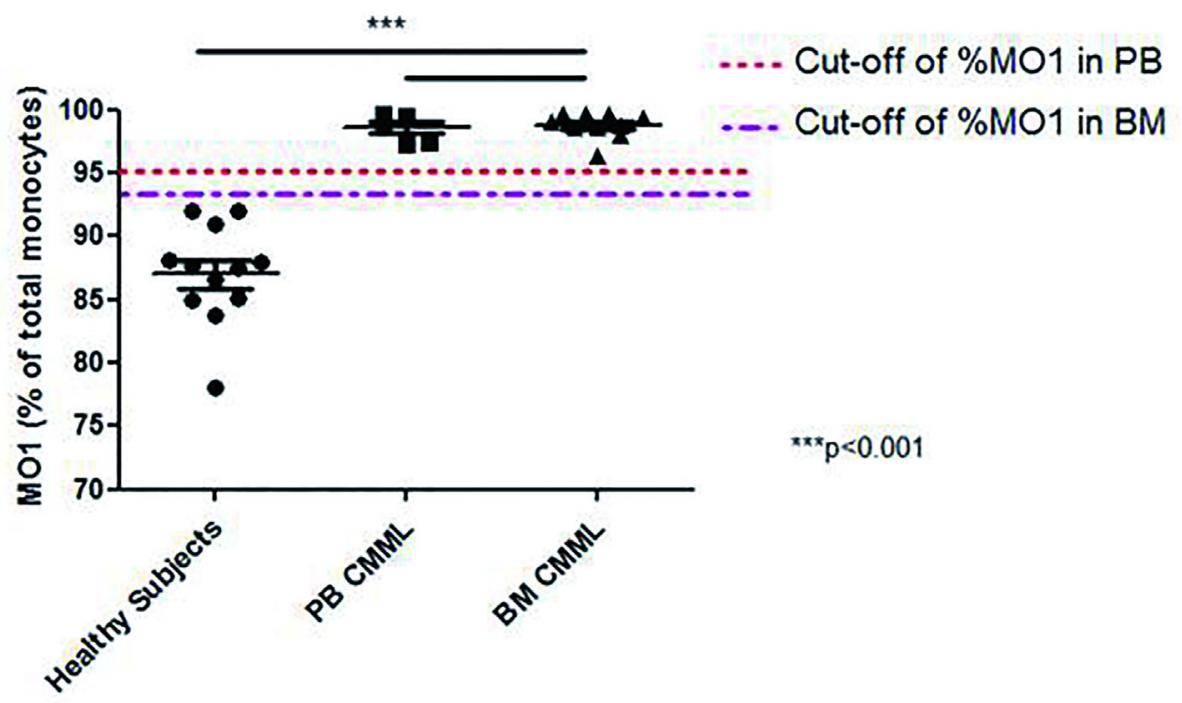

B

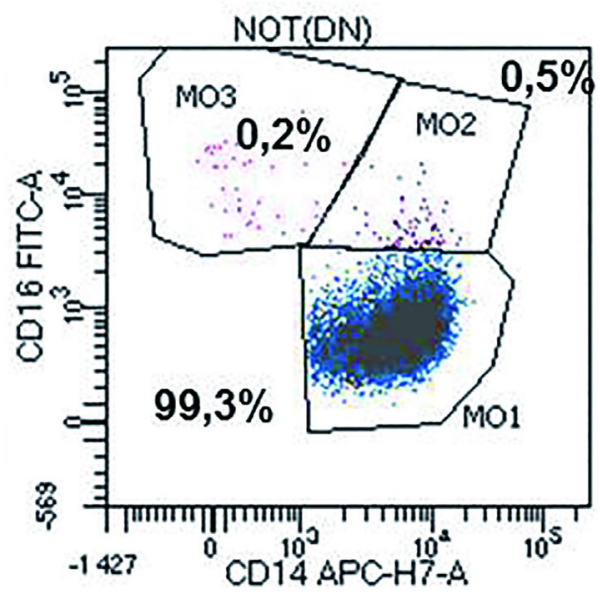

48 Hours

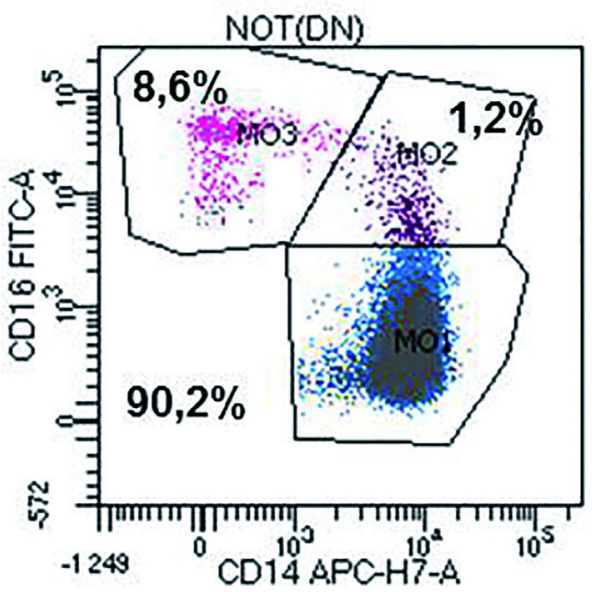

FIGURE 3 | Abnormal repartition of monocyte subsets in chronic myelomonocytic leukemia (CMML). Evaluation of the MO1 percentage cut-off subset in peripheral blood (PB) $(n=5)$ and bone marrow (BM) $(n=10)$ in the CMML group (A). The MO1 percentage cut-off points were compared with the threshold set $\left(1 \times 10^{9} / L\right)$ for the PB monocytosis. The differences between the values obtained in the MO1 percentage in BM and PB for the CMML group compared with those detected in the healthy subjects were statistically significant $\left({ }^{\star * \star} p<0.001\right.$; Fisher test). Representative example for the differences observed in the distribution of monocytes subset when the test was performed at 24 and $48 \mathrm{~h}$ after sample harvesting. The monocytes subsets are expressed as a percentage of total monocytes (B). 
BM (100\% specificity, $100 \%$ sensitivity, $p<10^{-4}, \chi^{2}$ test, Youden index $=1$ ) (Figure 3A). Using these cut-offs, the MFC screening test was consistent with CMML diagnosis in 100\% (15/15) of the cases evaluated in BM samples. The MFC results were in concordance with BM cytology, karyotype, and molecular biology. In $100 \%$ of CMML cases, the MO2 percentage was $<2 \%$, whereas in reactive monocytosis cases it was always $>2 \%(p<0.001)$. Four reactive monocytosis cases presented high percentage of blood MO1 monocytes ( $>95 \%$ ), but with $\mathrm{MO} 2$ percentage $>2 \%$. In addition, the monocyte subpopulations screening by MFC was performed in two AML NPM1 ${ }^{+}$patients in remission, showing a persistent monocytosis and dysplastic signs in BM evaluation, after consolidation therapy in one case, and after allogeneic stem cell transplantation for the second case. In both cases, an increased level

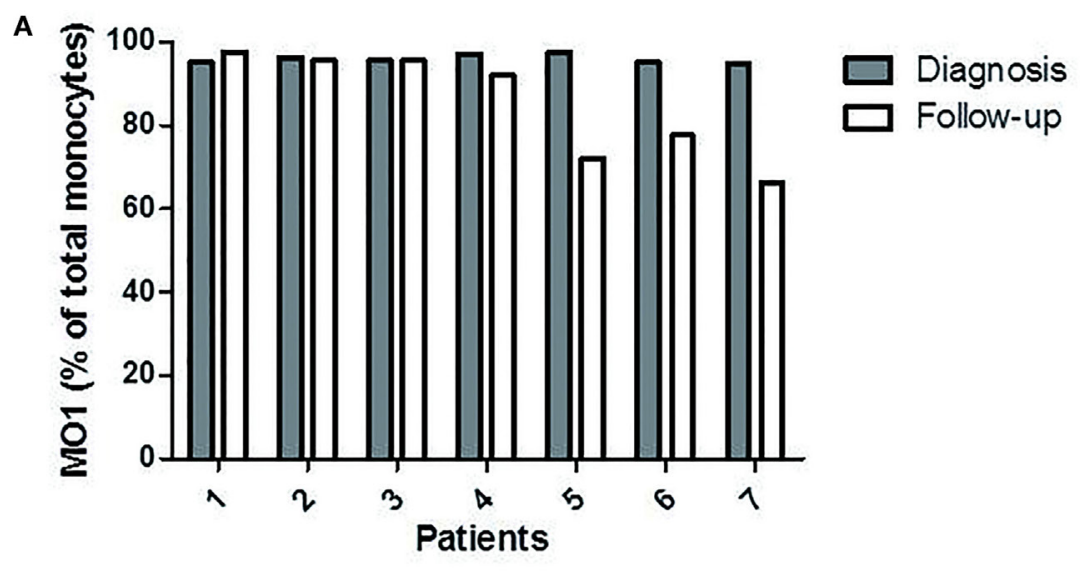

B



Follow-up disease

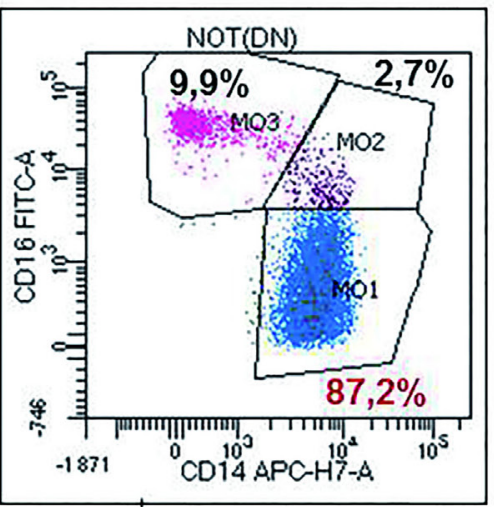

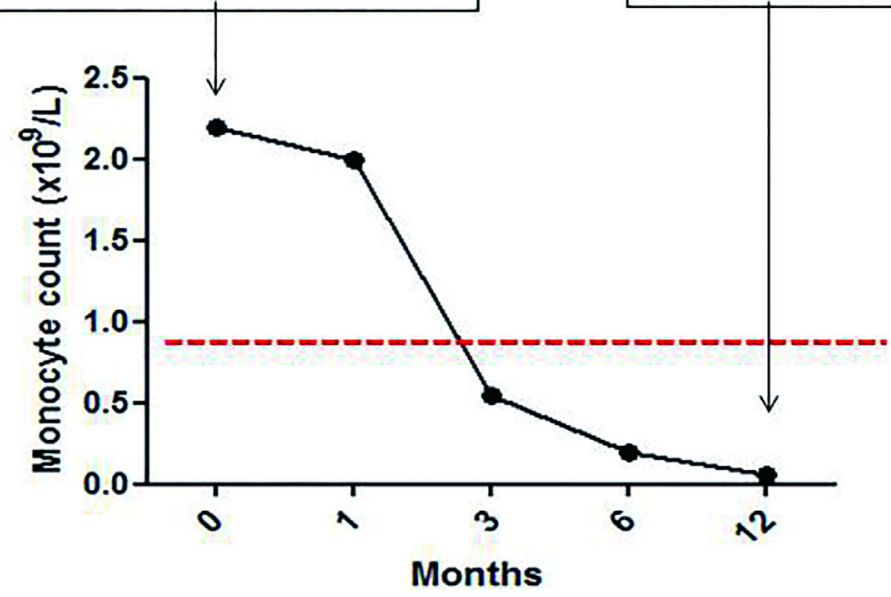

FIGURE 4 | The profile of the monocytes subsets during chronic myelomonocytic leukemia (CMML) follow-up. Relative frequencies of the MO1 subsets at the diagnosis point (dark bar) versus follow-up (white bar) ( $n=7 \mathrm{CMML}$ patients). MO1 are expressed as a percentage of total monocytes (A). Representative example for the evolution of \% MO1 classical monocytes during the follow-up in a CMML case with good response to the hypomethylating agents therapy (B). 
of MO1 monocytes (>96\%) was detected by MFC. NGS revealed, in these cases, respectively, a TET2 and an IDH2 mutation.

\section{Monocyte Subset Profile During Follow-Up}

The monocytes profile evaluation was performed in seven CMML cases during hypomethylating agents (HMA) treatment (4 PB and 3 BM samples). A significant decrease in the percentage of MO1 monocytes was observed in four patients (patients 4, 5, 6, and 7; Figure 4A) (mean of the group 77.05\% \pm 0.14 for $\mathrm{MO} 1,10 \% \pm 0.03$ for $\mathrm{MO} 2$, and $12.20 \% \pm 0.12$ for MO3) along with a return to normal values of monocyte counts $\left(<1 \times 10^{9} / \mathrm{L}\right)$. Figure 4B shows a representative example of a good response to HMA therapy. A persistence of CMML monocytosis was observed in three patients (patients 1, 2, and 3) along with an increased percentage in MO1 $>95 \%$ (mean of $96.3 \% \pm 0.02$ for $\mathrm{MO} 1,2.5 \% \pm 0.006$ for $\mathrm{MO} 2$, and $2.3 \% \pm 0.01$ for MO3) (Figure 4A). The MFC results were confirmed in patients 1-3 by cytological analysis, and persistence of dysplastic signs in BM myeloid cells was reported.

\section{DISCUSSION}

With overlapping MDS and MPN features, CMML displays a complex biological and clinical heterogeneity, which is not reflected by the mutational landscape (15). This is illustrated by the absence of disease-relevant mutations that can recapitulate the CMML phenotype in mouse models (15).

The exhaustive studies evaluating BM myeloblasts, monocytes, and granulocytes showed the existence of multiple MFC abnormalities in myeloid compartments in CMML, which are closer to MDS than MPN, but also unspecific for CMML $(5,16)$. However, evaluation by MFC of all myeloid compartments in BM is a complex, expensive, time-consuming procedure. Therefore, MFC testing of PB monocyte subsets using a limited number of antibodies in one single tube is a promising tool for the differential diagnosis between CMML and reactive monocytosis (11). We confirm that this method is rapid and efficient in $\mathrm{PB}(\mathrm{NPV}=1$, $\mathrm{PPV}=0.8)$ and in $\mathrm{BM}(\mathrm{NPV}=1, \mathrm{PPV}=1)$. Significant thresholds for MO1 monocytes in CMML were observed in $\mathrm{PB}(>95 \%)$ and $\mathrm{BM}(>93 \%)$ was not previously described. The addition of MO2 evaluation to that of MO1 allowed us to reach the same levels of sensitivity and specificity in $\mathrm{PB}$ as in $\mathrm{BM}$, as four cases with a percentage of $\mathrm{MO} 1>95 \%$ were reassigned to a diagnosis of reactive monocytosis.

The major drawback of this test is the choice of thresholds between MO1, MO2, and MO3 subpopulations. The previously published studies used an automated clustering algorithm called spanning-tree progression analysis of density-normalized events (SPADE) to confirm the gating strategy (Cytobank software) in order to avoid gaiting errors $(11,17)$. Therefore, in order to improve our gating strategy, an evaluation of some cases using the Infinicyt software (version 1.8) was carried out. Although, for healthy controls and reactive monocytosis the automatic population separator analysis helped for better identification of the
MO1, MO2, and MO3. However, this method was not efficient in CMML cases (data not shown). We also studied the stability of monocyte subset distribution during the sample storage. We showed that the delay between sampling and testing should not exceed $24 \mathrm{~h}$ in order to avoid the decrease in MO1 and the increase in $\mathrm{MO} 2$ subsets. This pre-analytical issue has not been described in previous studies, but can explain false negative results and a lack of specificity.

A return to normal values of monocytic subtypes could be used as a simple marker in the disease follow-up. Our preliminary data revealed a normal pattern of monocytes distribution in CMML patients in remission, but further studies are needed to evaluate the usefulness of this test for disease monitoring. Three patients who showed persistent abnormal monocyte distribution had unfavorable molecular prognostic factors (i.e., ASXL1, TET2, SRSF2, and IDH2 mutations) which correlated with poor response to HMA therapy.

Furthermore, this test was performed in two AML patients in remission, who exhibited constant monocytosis during treatment. MFC testing revealed an increase in MO1 subpopulation $>96 \%$ which was in line with NGS findings showing a TET2 and an $I D H 2$ mutation, respectively. Mutations in epigenetic modifying enzymes, such as TET2 and IDH2, are highly prevalent in CMML (18-20) and associated with DNA hypermethylation $(21,22)$. The diagnosis of secondary CMML was set in these cases by corroborating evidence with other biological tests and clinical manifestations.

In conclusion, using only blood monocytosis criteria, the diagnosis of CMML is difficult when dysplasia is not evident. BM cell karyotype may comfort CMML diagnosis, but anomalies are observed only in a minority of cases (less than 30\%) and are not specific to this disease. Our findings suggest a hierarchy of biological tests for CMML diagnosis, first relying on the MFC test of blood samples to exclude non-CMML patients from further invasive procedures. The NGS test is in search of recurrently mutated genes in myeloid malignancies and a complete evaluation including BM exploration could be reserved for patients with abnormal MFC results.

\section{ETHICS STATEMENT}

All patients gave informed consent according to the institutional procedures.

\section{AUTHOR CONTRIBUTIONS}

TP performed the experiments. TP, CA, PF-G analyzed data, wrote the article, created the tables, figures, and gave final approval. PN, ST contributed to data analysis and reviewed the article. ET, DG, LC reviewed the article and gave final approval.

\section{ACKNOWLEDGMENTS}

We thank BD Biosciences for providing monoclonal antibodies. 


\section{REFERENCES}

1. Bacher U, Haferlach T, Schnittger S, Kreipe H, Kröger N. Recent advances in diagnosis, molecular pathology and therapy of chronic myelomonocytic leukemia. Br J Haematol (2011) 153:149-67. doi:10.1111/j.1365-2141.2011. 08631.x

2. Orazi A, Bennett JM, Germing U, Brunning RD, Bain BJ, Thiele J. Chronic myelomonocytic leukaemia. In: Swerdlow SH, Campo E, Harris NL, Jaffe ES, Pileri SA, Stein H, et al., editors. World Health Organization Classification of Tumours of Haematopoietic and Lymphoid Tissues. Lyon: IARC Press (2008). p. 76-9.

3. Itzykson R, Solary E. An evolutionary perspective on chronic myelomonocytic leukemia. Leukemia (2013) 27:1441-50. doi:10.1038/leu.2013.100

4. Vardiman JW, Thiele J, Arber DA, Brunning RD, Borowitz MJ, Porwit A, et al. The 2008 revision of the World Health Organization (WHO) classification of myeloid neoplasms and acute leukemia: rationale and important changes. Blood (2009) 114:937-51. doi:10.1182/blood-2009-03-209262

5. Shen Q, Ouyang J, Tang G, Jabbour EJ, Garcia-Manero G, Routbort M, et al. Flow cytometry immunophenotypic findings in chronic myelomonocytic leukemia and its utility in monitoring treatment response. Eur J Haematol (2015) 95(2):168-76. doi:10.1111/ejh.12477

6. Van DJJM, Orfao MCEVJA. Generic Methods and Means for Monitoring Disruption of Tissue Homeostasis in the Total Body. Google Patents US 2014/0024019 Al (2014). Available from: https://patents.google.com/patent/ US20140024019A1/en (Accessed: January 23, 2014).

7. Ziegler-Heitbrock L, Ancuta P, Crowe S, Dalod M, Grau V, Hart DN, et al. Nomenclature of monocytes and dendritic cells in blood. Blood (2010) 116:e74-80. doi:10.1182/blood-2010-02-258558

8. Cros J, Cagnard N, Woollard K, Patey N, Zhang SY, Senechal B, et al. Human CD14dim monocytes patrol and sense nucleic acids and viruses via TLR7 and TLR8 receptors. Immunity (2010) 33:375-86. doi:10.1016/j. immuni.2010.08.012

9. Wong KL, Tai JJ-Y, Wong W-C, Han H, Sem X, Yeap WH, et al. Gene expression profiling reveals the defining features of the classical, intermediate, and nonclassical human monocyte subsets. Blood (2011) 118:e16-31. doi:10.1182/ blood-2010-12-326355

10. Zawada AM, Rogacev KS, Rotter B, Winter P, Marell RR, Fliser D, et al. SuperSAGE evidence for CD14+CD16+ monocytes as a third monocyte subset. Blood (2011) 118:e50-61. doi:10.1182/blood-2011-01-326827

11. Selimoglu-Buet D, Wagner-Ballon O, Saada V, Bardet V, Itzykson R, Bencheickh L, et al. Characteristic repartition of monocyte subsets as a diagnostic signature of chronic myelomonocytic leukemia. Blood (2015) 125:3618-26. doi:10.1182/blood-2015-01-620781

12. Arber DA, Orazi A, Hasserjian R, Thiele J, Borowitz MJ, Le Beau MM, et al. The 2016 revision to the World Health Organization classification of myeloid neoplasms and acute leukemia. Blood (2016) 127:2391-405. doi:10.1182/ blood-2016-03-643544
13. Kalina T, Flores-Montero J, van der Velden VHJ, Martin-Ayuso M, Böttcher S, Ritgen M, et al. EuroFlow standardization of flow cytometer instrument settings and immunophenotyping protocols. Leukemia (2012) 26:1986-2010. doi:10.1038/leu.2012.122

14. Shaffer LG, McGowan-Jordan J, Schmid M. An International System for Human Cytogenetic Nomenclature (ISCN 2013). Karger (2013).

15. Ball M, List AF, Padron E. When clinical heterogeneity exceeds genetic heterogeneity: thinking outside the genomic box in chronic myelomonocytic leukemia. Blood (2016) 128:2381-7. doi:10.1182/blood-2016-07-692988

16. Subira D, Font P, Villalon L, Serrano C, Askari E, Gongora E, et al. Immunophenotype in chronic myelomonocytic leukemia: is it closer to myelodysplastic syndromes or to myeloproliferative disorders? Transl Res (2008) 151:240-5. doi:10.1016/j.trsl.2008.03.001

17. Mukherjee R, Barman PK, Thatoi KP, Tripathy R, Das BK, Ravindran B. Non-classical monocytes display inflammatory features: validation in sepsis and systemic lupus erythematous. Nature (2015) 5:13886. doi:10.1038/ srep 13886

18. DiNardo CD, Jabbour E, Ravandi F, Takahashi K, Daver N, Routbort M. IDH1 and IDH2 mutations in myelodysplastic syndromes and role in disease progression. Leukemia (2015) 30:980-4. doi:10.1038/leu.2015.211

19. Itzykson R, Kosmider O, Renneville A, Gelsi-Boyer V, Meggendorfer M, Morabito $\mathrm{M}$, et al. Prognostic score including gene mutations in chronic myelomonocytic leukemia. J Clin Oncol (2013) 31:2428-36. doi:10.1200/JCO. 2012.47.3314

20. Malcovati L, Papaemmanuil E, Ambaglio I, Elena C, Galli A, Della Porta MG, et al. Driver somatic mutations identify distinct disease entities within myeloid neoplasms with myelodysplasia. Blood (2014) 124:1513-21. doi:10.1182/ blood-2014-03-560227

21. Figueroa ME, Skrabanek L, Li Y, Jiemjit A, Fandy TE, Paietta E. MDS and secondary AML display unique patterns and abundance of aberrant DNA methylation. Blood (2009) 114:3448-58. doi:10.1182/blood-2009-01200519

22. Meldi K, Quin T, Buchi F, Droin N, Sotzen J, Micol JB, et al. Specific molecular signatures predict decitabine response in chronic myelomonocytic leukemia. J Clin Invest (2015) 125(5):1857-72. doi:10.1172/JCI78752

Conflict of Interest Statement: The authors declare that the research was conducted in the absence of any commercial or financial relationships that could be construed as a potential conflict of interest.

Copyright (C) 2018 Picot, Aanei, Flandrin Gresta, Noyel, Tondeur, Tavernier Tardy, Guyotat and Campos Catafal. This is an open-access article distributed under the terms of the Creative Commons Attribution License (CC BY). The use, distribution or reproduction in other forums is permitted, provided the original author $(s)$ and the copyright owner are credited and that the original publication in this journal is cited, in accordance with accepted academic practice. No use, distribution or reproduction is permitted which does not comply with these terms. 